\title{
INSTITUTE OF RESEARGH ADVANCES
}

\section{IRA-International Journal of Technology e Engineering ISSN 2455-4480 Vol. 03 Issue 01 (April, 2016) Paper DOI: https://dx.doi.org/10.21013/jte.v3.nl.p3}

\section{An Algorithmic Approach towards Chest X-Ray Image Enhancement by Global Histogram Equalization}

\author{
G. Raghavendra Prasad \\ Assistant Professor \\ Amity University Chhattisgarh, Raipur, India.
}

\begin{abstract}
In medical imaging, the scope of image enhancement is highly challenging. Here digital chest $x$-ray image are taken in a spatial domain and enhancement of the image is done through histogram equalization method. Histogram equalization is a specific case of the more general class of histogram methods. Histogram Equalization works the best when applied to images with much higher color depth like continuos data or 16 bit gray scale images. In particular, the method can lead to better views of bone structure in X-ray images that are either over or under exposed. An algorithm is proposed to enhance the chest x-ray images using Global Histogram Equalization.
\end{abstract}

Keywords: Medical Imaging, GHE,Histogram

\section{Introduction:}

Consider a moment continuous function, and let the variable are represent the gray level of the taken chest $x$ ray image which is to be enhanced. In the beginning, we assume that $r$ has to be normalized to the interval $[0,1]$, with $r=0$ representing black and $r=1$ representing white. Later we consider a discrete formulation and allow pixel values to be in the interval[0,L-1].The entire operation can be expressed as $\mathrm{P}(\mathrm{M}(\mathrm{I}))$ where $\mathrm{I}$ is the original chest $\mathrm{X}$ ray image, $\mathrm{M}$ is histogram equalization mapping operation and $\mathrm{P}$ is a palette.This process shoots the global contrast of images taken when the information to be extracted or well represented is represented by close contrast coordinate values.By this fine tuning the intensities are better spread across on the histogram. Histogram Equalization method 
permits local contrast to gain a higher contrast without changing the global contrast and distributes most frequent intensity values equally.

IMAGE ACQUISITI ON $\rightarrow$ NOISE REMOVAL $\rightarrow$ MASKI NG $\rightarrow$ SHARPENI NG $\rightarrow$ MASKING

IMG RECONSTRUCTION

Fig 1: Schema of proposed work

\section{PROPOSED ALGORITHM:}

Step 1: Normalizing image intensity values of chest X-rays between $[0,1]$

Step 2: Depending upon the nature of image Hard or Soft thresholding is applied for attenuating the noise on the basis of input values.

Step 3: A Laplacian mask has been chosen in order to sharpen the image with reference to the neighboring pixels

$g(x, y)=f(x, y) \Delta \quad 2 f(x, y) / /$ center coefficient of the mask is negative

$$
\mathrm{f}(\mathrm{x}, \mathrm{y}) \quad \Delta{ }^{2} \mathrm{f}(\mathrm{x}, \mathrm{y})
$$

Input image $=\mathrm{f}(\mathrm{x}, \mathrm{y})$

Processed image $=\mathrm{g}(\mathrm{x}, \mathrm{y})$

Step 4: A $3 \times 3$ filter is used to emphasize horizontal edges using smoothing effect by approximating a vertical gradient.

Step 5: The smoothened gradient obtained by using an averaging filter of size $5 \times 5$

The two gradient images are scaled for display in the same manner as Laplacian images

Step 6: Image sharpening is done by adding original image with product of step4 and step5

Step 7: Histogram Equalization is performed over Step6 resultant image 
Step 8: Reconstruction of the image is accomplished.

\section{Implementation:}

Let $n_{I}$ be the number of occurrences of gray scale in a given chest xray image .The probability of an occurrence of a pixel of level I in the image is

$\mathrm{P}\left(\mathrm{x}_{\mathrm{i}}\right)=\mathrm{n}_{\mathrm{i}} / \mathrm{n}, i \in 0, \ldots L-1$

$\mathrm{L}$ being the total number of gray levels in the image, $\mathrm{n}$ being the total number of pixels in the image and $p$ being in fact the image's histogram, normalized to[0,1].

Let us also define $\mathrm{c}$ as cumulative distribution function corresponding to $\mathrm{p}$, defined by $c(i)=\sum_{j=0}^{i} p(x j)$

Also known as the image's accumulated normalized histogram.

We would like to create a transformation of the form $\mathrm{y}=\mathrm{T}(\mathrm{x})$ that will produce a level $\mathrm{y}$ for each level $\mathrm{x}$ in the original image, such that the cumulative probability function of $\mathrm{y}$ will be linearized across the value range. The transformation is defined by

$\mathrm{y}_{\mathrm{i}}=\mathrm{T}\left(\mathrm{x}_{\mathrm{i}}\right)=\mathrm{C}(\mathrm{i})$

Note that the Tmaps the levels into the domain of $0 \ldots 1$.In order to map the values back into their original domain,The following simple transformation needs to be applied on the result.

$$
y_{i}^{I}=y_{i} *(\max -\min )+\min
$$

GHE is based on the statistical information of the whole image .Let $\Omega$ subset of IR ${ }^{2}$ is the image domain and $\mathrm{u}: \Omega \rightarrow[\mathrm{a}, \mathrm{b}]$ be the given (Low contrast chest xray).Let $\mathrm{f}:[\mathrm{a}, \mathrm{b}] \rightarrow[\mathrm{c}, \mathrm{d}] \mathrm{be}$ a given increasing function. Then $\mathrm{v}:=\mathrm{f}(\mathrm{u})$ represents the stretched image. In order to distribute the gray levels equally to a new range from $[a, b]$, the probability density of each level must be obtained.

\section{Quality Measure:}

The quantitative measurement of the contrast improvement is often very difficult. Moreover here is no universal measure specifying both the objective and the subjective validity of the enhancement method .The contrast is usually defined as the difference in mean luminance between an object and its surroundings. In practice, there are many definitions of the contrast measure. When an image is composed of textured regions such as mammographic images, it seems more desirable to define the local contrast. 
The local contrast proposed by Gordan and Rangayyan was defined by the mean gray values in two rectangular windows centered on a current pixel(x,y). Beghdadi and Negrate proposed another definition of the local contrast based on local edge information of the image in order to improve the definition of Gordon and Rangayyan. This paper adopts the local contrast proposed by Baghdadi and Negrate in order to define a performance measure of enhancement.

For the objective evaluation of the contrast improvement, the proposed method was compared with three conventional enhancement methods such as the direct contrast enhancement, the adaptive histogram equalization, and the unsharp masking. The results were found quite significant.

\section{Conclusive Theory:}

There have been remarkable advances in conventional imaging over the past decade. Opportunities for direct computer aided detection of various lesions may enhance the radiologists' accuracy and improve efficiency. Recent techniques such as dual energy and temporal subtraction radiography show promise along with Digital tomosynthesis.In scientific imaging where spatial correlation is more important than the intensity of signal(such as separating DNA fragments of quantized length), the small signal to noise ratio usually hampers visual detection. Histogram Equalization provides better detect ability of fragment size distributions. To better appreciate the recent advances in chest radiography, we must first review some of the inheret challenges of the techniques, because these challenges have been the prime motivators behind most of the developments. These challenges include but are not limited to issues related to image area and patient body habitats, latitude and dynamic range of $x$ ray, transmission through chest, scattered radiation, overlap of anatomic structures and perceptual limitations.

\section{Reference:}

1. Digital Image Processing Using MATLAB 2nd Ed. by Gonzalez, Woods, and Eddins (C) 2009 .

2. Fundamentals of Digital Image Processing by Anil K. Jain. 